\title{
New Active Control Method Based on Using Multiactuators and Sensors Considering Uncertainty of Parameters
}

\author{
Babak Karimpour, ${ }^{1}$ Ali Keyhani, ${ }^{1}$ and Javad Alamatian ${ }^{2}$ \\ ${ }^{1}$ Department of Civil Engineering, Industrial University of Shahroud, Shahroud 3619995161, Iran \\ ${ }^{2}$ Department of Civil Engineering, Mashhad Branch, Islamic Azad University, Mashhad 9179156314, Iran \\ Correspondence should be addressed to Javad Alamatian; alamatian@mshdiau.ac.ir
}

Received 15 November 2013; Revised 23 February 2014; Accepted 23 February 2014; Published 31 March 2014

Academic Editor: Andreas Kappos

Copyright (c) 2014 Babak Karimpour et al. This is an open access article distributed under the Creative Commons Attribution License, which permits unrestricted use, distribution, and reproduction in any medium, provided the original work is properly cited.

\begin{abstract}
New approach is presented for controlling the structural vibrations. The proposed active control method is based on structural dynamics theories in which multiactuators and sensors are utilized. Each actuator force is modeled as an equivalent viscous damper so that several lower vibration modes are damped critically. This subject is achieved by simple mathematical formulation. The proposed method does not depend on the type of dynamic load and it could be applied to control structures with multidegrees of freedom. For numerical verification of proposed method, several criterions such as maximum displacement, maximum kinetic energy, maximum drift, and time history of controlled force and displacement are evaluated in two- , five- , and seven-story shear buildings, subjected to the harmonic load, impact force, and the Elcentro base excitation. This study shows that the proposed method has suitable efficiency for reducing structural vibrations. Moreover, the uncertainty effect of different parameters is investigated here.
\end{abstract}

\section{Introduction}

Smart structures are systems that can teach and protect themselves against the external excitation such as wind and earthquake. Analyzing and designing of smart structures is based on set of sciences including materials science, applied mechanics, electronics, biomechanics, and structural dynamics. In this procedure, maintaining the structural performance against the external hazards is a very important issue called control system. Many studies have been performed in the field of structural control. These methods can be categorized into three groups, that is, passive, semiactive, and active procedures [1]. Due to the simplicity, low cost of assembly and no need to the external power, the passive control systems are numerous. However, the constant control feature makes these systems fail during the earthquakes. In other words, these systems are designed to work only for a certain excitation and limited frequency bound.

The passive control system tries to remove the kinetic energy from the structure. Because of the mentioned constraints in passive algorithms, active control is highly regarded systems to cope with the earthquake. These techniques have suitable efficiency in different excitation, so that they could exactly sense and adopt the structural vibrations. To achieve this goal, each active control method is constructed based on algorithm, which verifies its efficiency and accuracy. The application of such systems began in 1989. In these systems an external power source is required so that this applied force affects the structural equilibrium equation. This applied force may lead to instable vibrations if the active control algorithm is not suitable.

Hence, the complexity, the calculations volume, the instability risk, and the uncertainty factor are some difficulties that arise from active control systems. It should be noted that good performance of active methods depends on some parameters such as the reliable algorithm and the suitable positions for both sensors and actuators [2-5].

There are several active control mechanism proposed by different researchers that deal with such subjects. In this way, Bayard and his coworkers present the $\mathrm{D}$-optimal design principle which is chosen by the maximum determinant of Fisher information matrix as the criteria function [6]. This paper 
simplifies the selected modes into a unitary form by a simple method, so that the suitable position of the piezoelectric elements is achieved. Moreover Kamada and his coworkers modeled a four-story building, which was controlled by piezoelectric actuators, utilizing different algorithms [7]. Such study shows that floor accelerations could be reduced up to seventy percent. The subject of determining the suitable position of the piezoelectric actuators was investigated by some other researchers. For example, Han and Lee present a controllable Grammian matrix for the smart composite plates, in which the maximum eigenvalue is used as performance function [8]. In this study, the genetic algorithm was utilized to find the effective locations of piezoelectric sensors and actuators. Moreover, Sadri and his coworkers presented some criteria for determining the optimal position of piezoelectric actuators using the controllability of modes [9]. In the other research, Gao and his coworkers considered a vibration suppression problem so that the total radiated acoustic power or acoustic potential energy is minimized [10]. They used genetic algorithm with immune diversity to evaluate the suitable positions of actuators. Simultaneously, Zhang and his coworkers invested a performance function based on maximizing the dissipated energy that arises from the control action [11]. According to this study, a float-encoded genetic algorithm was presented which is capable of solving this optimization problem. Cao and his coworkers used the element sensitivities of singular values to identify the suitable positions of actuators, based on running an optimization process [12]. By using topology optimization Kögl and Silva presented an approach to design the piezoelectric plates and shell actuators [13]. In this method, the optimization problem consists of distributing the piezoelectric actuators in such a way as to achieve a maximum output displacement in a given direction at a given point of the structure. Moreover, an experimental study on piezoelectric actuators was performed by Sethi and Song [14]. They controlled the vibrations of three-story frame, using a piezoelectric patch sensor and actuator. Also, they implemented pole placement modal mechanism to control all vibrations modes. Furthermore than using piezoelectric, there are other kinds of active control algorithms. For example, Song and his coworkers presented the active control mechanism for space truss, using a Lead zirconate Titanate stack actuator [15]. The common active control algorithms have been listed in Table 1, followed by the main idea used in each method [16]. Finally, the semiactive procedures are achieved based on modifying the passive control systems in combination with active mechanisms [17].

It should be noted that the common active control algorithms use the mathematical concepts such as the genetic algorithms, the Fuzzy logical approaches, the optimization techniques, and other mathematical theories. In these methods, the fundamental principles of structural dynamic which introduce the dynamic behavior of structures were ignored. For this reason, common active control schemes are consistent with structural behavior. The proposed method tries to solve this defect so that the new algorithm is achieved based on a well-known structural dynamics theory, that is, critical damping concept. Based on this theory and also using multiple actuators, a new method is presented here for active control of structures. For this purpose, some fundamental theories of structural dynamics are utilized so that the actuators are modeled as additional viscous dampers in dynamic equilibrium equation. This procedure leads to the actuators forces. Moreover proper positions of both actuators and sensors are determined by an innovate technique. Efficiency of the proposed control method is also evaluated by solving some numerical examples.

\section{The Proposed Active Control Concept Based on Multiactuators and Sensors}

Dynamic equilibrium equation of structure can be implemented with various methods such as the Hamilton principle [28]:

$$
\mathrm{MD}+\mathbf{C} \dot{\mathbf{D}}+\mathbf{S D}=\mathbf{P},
$$

where $\mathbf{M}, \mathbf{C}$, and $\mathbf{S}$ are mass, damping, and stiffness matrices of structure, respectively. Furthermore $\mathbf{P}$ and $\mathbf{D}$ are external force and the nodal displacement vectors, respectively. Also, super dots $(\cdot)$ denote differential with respect to time. In active control case, the dynamic equilibrium equation is incorporated into the following relationship:

$$
\mathbf{M D}+\mathbf{C D}+\mathbf{S D}+\mathbf{F}^{\mathrm{a}}=\mathbf{P},
$$

where $\mathbf{F}^{\mathbf{a}}$ is the equivalent actuator force vector which is generated from the active control mechanism. From the structural dynamics point of view, the structural vibrations damp in the lowest possible time if the structure behaves in the critical damping condition. This is the main concept used here for designing new active control procedure. To explain this idea, consider a multidegree of freedom structure i.e.q. In modal dynamic analysis, this structure has $q$ vibrations mode. For exposing the structure to behave in fully critical damping conditions, all vibration modes should be in critical damping conditions. From this point of view, $q$ actuators should be attached to structure which causes all elements of the actuator force vector, $\mathbf{F}^{\mathbf{a}}$, to be nonzero. It is clear that high number of actuators increases the cost of control process which is not suitable. To prevent this difficulty, few numbers of actuators, that is, $(n$ less than $q$ ) is used. Also, the effect of lower dynamic modes is more than higher modes. Therefore $n$ actuators forces are calculated so that $n$ lower modes oscillate in critical damping conditions. Based on the above discussion, the critical damping theory is utilized to determine the equivalent actuators forces. For this purpose, (2) is transformed to the modal space as follows:

$$
M_{i} \ddot{Z}_{i}+C_{i} \dot{Z}_{i}+S_{i} Z_{i}+\varphi_{i}^{T} F^{a}=\varphi_{i}^{T} P \quad i=1,2, \ldots, q,
$$

where $M_{i}, C_{i}$, and $S_{i}$ are mass, damping, and stiffness of $i$ th modal coordinates, that is, $Z_{i}$, respectively. Also, $q$ is the number of degrees of freedom and $\varphi_{i}$ is the $i$ th mode shape vector of free vibration of the structure. If the mode's rank in (3) increases, its effect on dynamic response decreases. Therefore, the primary mode has the highest effect on the dynamic response compared with other vibrations modes. 
TABLE 1: Common Active Control algorithms.

\begin{tabular}{ll}
\hline Active control method & Main concept \\
\hline Linear optimal control & Minimize the performance index (Yang 1975 [18]) \\
Pole assignment technique & Minimize the performance index (Abdel-Rohman and Leipholz 1978 [19]) \\
Independent modal space control & Minimize the modal control force (Meirovitch and Oz 1980 [20]) \\
Instantaneous optimal control & Control by minimizing the energy function at each instant of time (Yang et al. 1987 [21]) \\
Bounded state control & Keeping the range of responses allowed (Reinhorn et al. 1987 [22]) \\
Nonlinear control & Minimizing higher-order function (Wu et al. 1995 [23]) \\
Generalized feedback & Control force will be a function of a displacement, velocity, and acceleration (Yang et al. 1991 [24]) \\
Sliding mode control (SMC) & Creating the sliding surface (Yang et al. 1994 [25]) \\
Time delay compensation & Enter the time delay between the response and performance of control (Abdel-Rohman 1985 [26]) \\
Database and rule base & Active control using neural network and fuzzy logic (Tani et al. 1998 [27]) \\
Genetic algorithm & Active control using genetic algorithm (Akutagawa et al. 2004 [1]) \\
\hline
\end{tabular}

This principle is utilized to obtain the equivalent actuators forces. Since there are $n$ actuators, attached to the structure, the modal equations could be written as follows:

$$
\begin{aligned}
& M_{i} \ddot{Z}_{i}+C_{i} \dot{Z}_{i}+S_{i} Z_{i}+\varphi_{k i} F_{k}^{a}+\varphi_{j i} F_{J}^{a} \\
& \quad+\cdots+\varphi_{r i} F_{r}^{a}=\varphi_{i}^{T} P, \quad i=1,2, \ldots, n,
\end{aligned}
$$

where $\varphi_{k i}$ is the $k$ th element of the $i$ th modal shape vector and $F_{k}^{a}$ is the equivalent actuator force attached to $k$ th degree of freedom. The actuators act as additional viscous dampers. This is a model used in the mathematical formulation of the proposed active control method. This model leads to an effective actuator's force which has suitable compatibility with structural behavior. It should be emphasized that modeling the actuator as an equivalent viscous damper does not mean that the actuator's force should be applied to the structure by viscous dampers. In other words, any device which produces such forces is suitable for using in the practical cases. Therefore, the proposed algorithm only presents/calculates the suitable value of the actuator's force in each time of dynamic analysis. Then, this force could be generated by any device of the power source such as piezoelectric. In the following, (4) is transformed to the following relationship:

$$
M_{i} \ddot{Z}_{i}+C_{i}^{*} \dot{Z}_{i}+S_{i} Z_{i}=\varphi_{i}^{T} P,
$$

where $C_{i}^{*}$ is the $i$ th equivalent coordinate damping which is formulated based on both natural structural damping and the effect of the actuators forces:

$$
\begin{array}{r}
C_{i}^{*}=C_{i}+\left(\frac{\varphi_{k i}}{\dot{Z}_{i}}\right) F_{k}^{a}+\left(\frac{\varphi_{j i}}{\dot{Z}_{i}}\right) F_{j}^{a}+\cdots+\left(\frac{\varphi_{r i}}{\dot{Z}_{i}}\right) F_{r}^{a} \\
i=1,2, \ldots, n .
\end{array}
$$

If the equivalent damping coordinates are equal to the critical damping, the structural oscillations damp in the lowest time:

$$
C_{i}^{*}=2 M i \omega_{i}, \quad i=1,2, \ldots, n,
$$

where $\omega_{i}$ is the $i$ th natural frequency of the structure. It is clear that using $n$ actuators lead to $n$ unknown actuators forces which should be determined at each second. For this purpose, (7) presents a system of equations which could be solved at each analysis time. In the case of existing one actuator substituting (6) into (7) leads to the following result:

$$
F_{k}^{a}=\frac{\left\{2 M_{1} \omega_{1} \dot{Z}_{1}-C_{1} \dot{Z}_{1}\right\}}{\varphi_{k 1}} .
$$

Equation (8) is completely consistent with the results presented by Alamatian and Rezaeepazhand [29] in which the active control process is formulated based on using only one actuator. Therefore, the proposed method is much more general than existing methods, so that the structural vibrations could be controlled by several actuators. In the case of structural control with two actuators, (7) leads to the below set of equations:

$$
\begin{aligned}
& C_{1}^{*}=C_{1}+\left(\frac{\varphi_{k 1}}{\dot{Z}_{1}}\right) F_{k}^{a}+\left(\frac{\varphi_{j 1}}{\dot{Z}_{1}}\right) F_{j}^{a}=2 M_{1} \omega_{1} \\
& C_{2}^{*}=C_{2}+\left(\frac{\varphi_{k 2}}{\dot{Z}_{2}}\right) F_{k}^{a}+\left(\frac{\varphi_{j 2}}{\dot{Z}_{2}}\right) F_{j}^{a}=2 M_{2} \omega_{2},
\end{aligned}
$$

where $k$ and $j$ are two degrees of freedom which actuators are attached to them. By solving system (9), the two actuators forcesare obtained:

$$
\begin{aligned}
F_{k}^{a}= & \left(\left\{2 M_{2} \omega_{2} \dot{Z}_{2}-C_{2} \dot{Z}_{2}\right.\right. \\
& \left.\left.-\left(\frac{\varphi_{j 2}}{\varphi_{j 1}}\right) \dot{Z}_{1}\left(2 M_{1} \omega_{1}-C_{1}\right)\right\}\right) \\
& \times\left(\left\{\varphi_{k 2}-\left(\frac{\varphi_{j 2}}{\varphi_{j 1}}\right) \varphi_{k 1}\right\}\right)^{-1} \\
F_{j}^{a}= & 2 M_{1} \omega_{1} \frac{\dot{Z}_{1}}{\varphi_{j 1}}-C_{1} \frac{\dot{Z}_{1}}{\varphi_{j 1}}-\varphi_{K 1} \frac{F_{k}^{a}}{\varphi_{j 1}} .
\end{aligned}
$$

Similar approach could be utilized to formulate the proposed active control method with three actuators. In this case, the 
first three damping coordinates are equal to their corresponding critical values, which leads to the following system of equations:

$$
\begin{aligned}
& C_{1}^{*}=C_{1}+\left(\frac{\varphi_{k 1}}{\dot{Z}_{1}}\right) F_{k}^{a}+\left(\frac{\varphi_{j 1}}{\dot{Z}_{1}}\right) F_{j}^{a}+\left(\frac{\varphi_{l 1}}{\dot{Z}_{1}}\right) F_{l}^{a}=2 M_{1} \omega_{1} \\
& C_{2}^{*}=C_{2}+\left(\frac{\varphi_{k 2}}{\dot{Z}_{2}}\right) F_{k}^{a}+\left(\frac{\varphi_{j 2}}{\dot{Z}_{2}}\right) F_{j}^{a}+\left(\frac{\varphi_{l 2}}{\dot{Z}_{2}}\right) F_{l}^{a}=2 M_{2} \omega_{2} \\
& C_{3}^{*}=C_{3}+\left(\frac{\varphi_{k 3}}{\dot{Z}_{3}}\right) F_{k}^{a}+\left(\frac{\varphi_{j 3}}{\dot{Z}_{3}}\right) F_{j}^{a}+\left(\frac{\varphi_{l 3}}{\dot{Z}_{3}}\right) F_{l}^{a}=2 M_{3} \omega_{3},
\end{aligned}
$$

where $k, j$, and $l$ are the three degrees of freedom; actuators are attached to them. Based on proposed method the actuators forces can be updated at any second of analysis just by solving a system of equations. There are some unknown parameters in previous systems of equations, that is, (9) and (11), such as coordinate's velocities $\left(\dot{Z}_{i}\right)$. Based on the structural dynamics theory, modal coordinate velocities depend on the nodal velocities. The coefficient of proportionality is elements of inverse modal shapematrix.

Consider

$$
\dot{Z}_{i}=\sum_{j=1}^{q} \varphi_{i j}^{\text {inv }} \dot{D}_{j}
$$

where $\varphi_{i j}^{\text {inv }}$ is $i j$ th element of the inverse modal shape matrix. To determine the modal velocities, it is necessary to determine both number of sensors and their locations. By increasing number of sensors, the accuracy of modal velocities increases. For example, if there are $m$ sensors attached to the structure, (12) can be written as follows:

$$
\dot{Z}_{i}=\varphi_{i L}^{\text {inv }} \dot{D}_{L}+\varphi_{i M}^{\text {inv }} \dot{D_{M}}+\cdots+\varphi_{i N}^{\text {inv }} \dot{D_{N}}
$$

Here, $L, M, \ldots, N$ are degrees of freedoms, sensors attached to them. It is clear that the proposed method presents the actuator's forces by solving a set of simultaneous equations in each time step. The dimension of this set of equation is equal to the number of actuators. Since number of actuators, attached to the structure, is finite (for practical cases), the dimension of the obtained set of simultaneous equations is quite small so that it solves in a small amount of time, compared with other calculations. Therefore, the required time, spent for calculating the actuator's forces is negligible. In other words, the proposed algorithm could run in real-time fast enough so that it can be utilized for active control of the realistic structures.

\section{The Proper Actuator and Sensor Locations}

In this section, the suitable degrees of freedoms actuators and sensors could be attached to them are evaluated. Here fundamental structural dynamics theories are utilized. For example the first mode usually has the highest portion in dynamic response. Therefore, degrees of freedom with high effect in the first mode are suitable for attaching actuators.
In other words, the proper degrees of freedom for actuator locations are those which their corresponding values in the first mode shape are higher than others. For determining the sensors locations, (13) is considered. The main criterion utilized for judgment about sensors locations is that reliable values of $\dot{Z}_{I}$ are obtained. These quantities correspond to both nodal velocities and elements of $\varphi^{-1}$. The nodal velocities are sensed from the dynamic structural response and will be unknown. The only available parameters are nodal velocity coordinates and elements of inverse of modal shape matrix. The proper sensor locations are determined based on those quantities so that sensors are attached to the degrees of freedom with higher corresponding values in the first row of the inverse matrix of mode shape. For example, in the case of two sensors, they should be installed in degrees of freedom associated with the two highest elements in the first row of the inverse matrix of mode shape. Since there are two sensors in the smart structure, the first and second modal coordinate's velocity can be calculated as follows:

$$
\begin{aligned}
& \dot{Z}_{1}=\varphi_{1 L}^{\text {inv }} \dot{D}_{L}+\varphi_{1 M}^{\text {inv }} \dot{D_{M}} \\
& \dot{Z}_{2}=\varphi_{2 L}^{\text {inv }} \dot{D}_{L}+\varphi_{2 M}^{\text {inv }} \dot{D_{M}},
\end{aligned}
$$

where $\varphi_{1 L}$ and $\varphi_{2 L}$ are $L$ th element in the first and second row of the inverse modal shape matrix. Also, $\dot{D}_{L}$ and and $\dot{D_{M}}$ are the velocity of $L$ th and $M$ th degree of freedom which the sensor are attached to them.

\section{The Proposed Active Control Algorithm}

To verify the proposed new active control method, some numerical dynamic analyses are performed. For this purpose, the analysis time is divided into limited number of time steps. In each time step, numerical time integration scheme will be utilized to achieve structural responses. Here, Newmark method with linear acceleration is used. The main steps of proposed active control process are as follows.

(a) Set $n=0$ and select the time step of dynamic analysis.

(b) Construct the stiffness, mass, and damping matrices.

(c) Determine the actuators and sensors locations using modal shape matrix and its inverse.

(d) Calculate the modal velocities.

(e) Solve set of equation to determine the actuators forces.

(f) Compute the displacements vector of the current time steps using Newmark method with linear acceleration [30].

(g) Calculate the structural velocity vector based on the Newmark method [30].

(h) Calculate the acceleration vector of $n$th time step by solving the following linear system:

$$
\mathbf{M D}=\mathbf{P}-\mathbf{C} \dot{\mathbf{D}}-\mathbf{S D}-\mathbf{F}^{\mathbf{a}} .
$$

(i) Set $n=n+1$. 


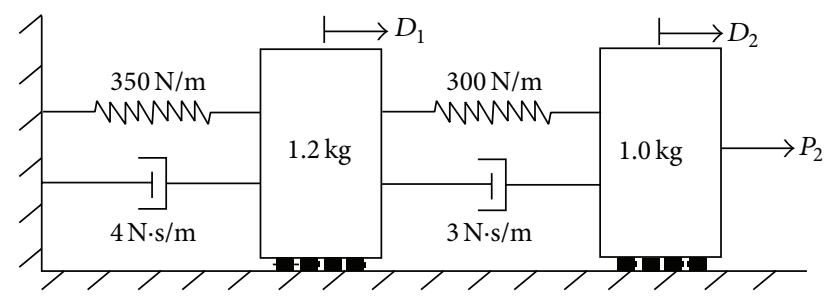

FIgURE 1: Two degrees of freedom system.

TABLE 2: Suitable sensor and actuator locations.

\begin{tabular}{lccc}
\hline Degree of Freedom & $\varphi_{1 L}^{\text {inv }}$ & $\varphi_{k 1}$ & Optimum Control Case \\
\hline 1 & 0.5831 & 0.5113 & S2-A2 \\
2 & 0.8167 & 0.8594 & \\
\hline
\end{tabular}

(j) If $n<n_{\max }$ go to (d).

(k) Print the results and end.

\section{Numerical Study}

To verify the validity of the proposed active control method, some numerical examples are presented. For this purpose, the suggested active control process is combined with numerical dynamic analysis methods, that is, Newmark integration using linear acceleration scheme.

5.1. Two Degree of Freedom System. Figure 1 shows a linear two-DOF system, subjected to impact load that is, $p_{2}$ defined as follows:

$$
P_{2}= \begin{cases}\frac{1}{\Delta t}, & t=\Delta t \\ 0, & \text { otherwise }\end{cases}
$$

where $\Delta t$ is the time step. The procedures, which leads to the optimum sensor and actuators locations, are summarized in Table 2. By applying the proposed method, the second degree of freedom is the suitable location for attaching both the actuator and sensor, that is, S2-A2, due to its highest corresponding values of $\varphi_{12}^{\text {inv }}=0.8167$ and $\varphi_{21}=0.8594$.

After determining the sensor and actuators locations, the proposed active control method is applied to this structure. Figures 2 and 3 show the displacement time responses of the first and second degrees of freedom for different control process, respectively. It is clear that all control methods are stable. On the other hand, the proposed method reduces the vibrations amplitude of both degrees of freedom in a short time. Therefore, the suggested process has suitable efficiency in vibration control. If the actuator and sensor are attached to the first degree of freedom (case S1-A1), the efficiency of the control process is reduced. Also, the control case S1A2 has lower efficiency than the two other cases. In fact S2-A1 is incapable of controlling the system. This subject clearly proves that the proposed algorithm for determining the actuator and sensors locations works property.

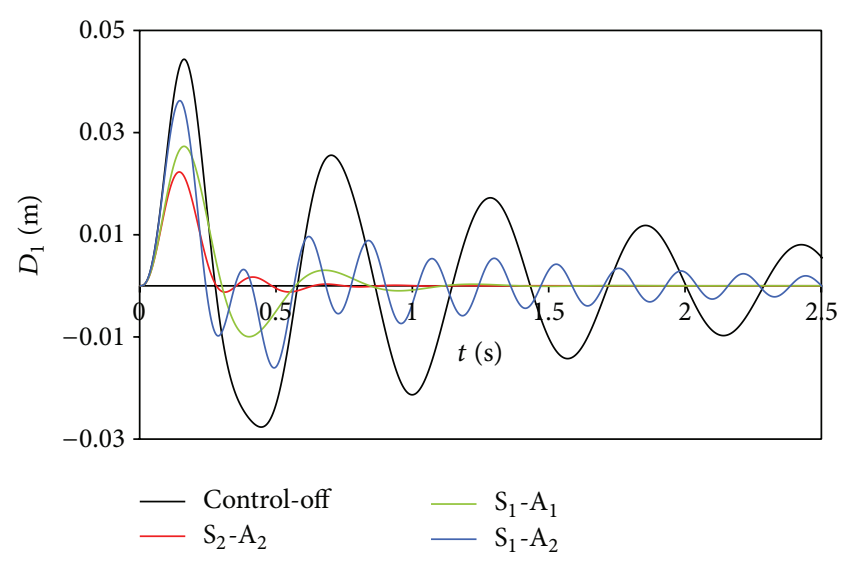

FIgURE 2: The displacement-time response to the first degree of freedom of 2D system.

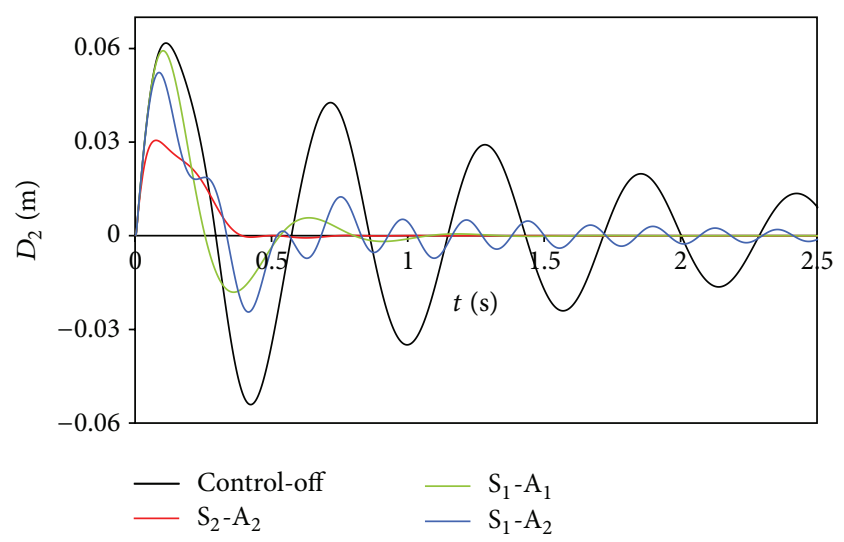

FIgURE 3: The displacement-time response to the second degree of freedom of $2 \mathrm{D}$ system.

5.2. Five Story Shear Building. Figure 4 shows a five-story shear building, modeled by lumped mass and lateral stiffness (five horizontal degrees of freedom). This structure is analyzed in two different cases. In the first analysis, a harmonic load that is, $p(t)=50 \sin (10 t)$, is applied to the fifth-story building. The second analysis is performed when the structure is excited by the Elcentro ground acceleration record.

In both analyses, the damping ratio of $5 \%$ for the first mode is assumed for constructing the Rayleigh damping matrix with two factors [31]. To control the vibrations of the structure, the location of the sensors and actuators are determined based on the proposed algorithm. Table 3 presents the details which lead to the optimum locations of sensors and actuators. Using the results presented in Table 3, the various control algorithms with regard to the number of actuators and sensors have been inserted in Table 4 .

Three factors are considered in numerical evaluation of the proposed method: the maximum displacements of stories, the maximum kinetic energy of the structure, and the maximum drift. Table 5 shows the maximum structural displacement for the harmonic load. 


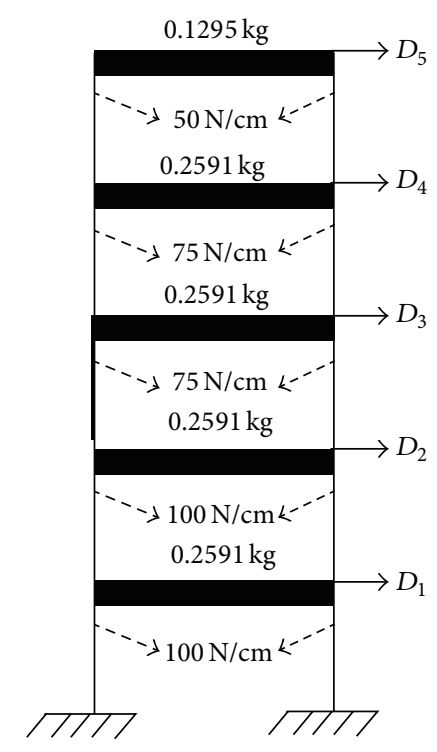

FIGURE 4: Five-story shear building.

TABLE 3: The actuator and sensor locations for the five-story shear building.

\begin{tabular}{lcccc}
\hline $\begin{array}{l}\text { Degree of } \\
\text { freedom }\end{array}$ & $\varphi_{K 1}$ & $\varphi_{1 L}^{\text {inv }}$ & $\begin{array}{c}\text { Priority of the } \\
\text { actuator installation }\end{array}$ & $\begin{array}{c}\text { Priority of the } \\
\text { sensor installation }\end{array}$ \\
\hline 1 & 0.1565 & 0.1922 & 5 & 5 \\
2 & 0.2991 & 0.3673 & 4 & 4 \\
3 & 0.4541 & 0.5576 & 3 & 2 \\
4 & 0.5555 & 0.6821 & 2 & 1 \\
5 & 0.6093 & 0.3741 & 1 & 3 \\
\hline
\end{tabular}

TABLE 4: Different control cases for the five-story shear building.

\begin{tabular}{ll}
\hline Mode & Characteristics of the control system \\
\hline Control off & $\begin{array}{l}\text { Structure without control system } \\
\text { Structure with one actuator (degree of freedom of 5) } \\
\text { and one sensor (degree of freedom of } 4 \text { ) }\end{array}$ \\
$\mathrm{A}_{5}-\mathrm{S}_{4}$ & Structure with 2 actuators and one sensor \\
$\mathrm{A}_{5,4}-\mathrm{S}_{4}$ & Structure with 3 actuators and 1 sensor \\
$\mathrm{A}_{5,4,3}-\mathrm{S}_{4}$ & Structure with 3 actuators and 2 sensor \\
$\mathrm{A}_{5,4,3}-\mathrm{S}_{4,3}$ & Structure with 3 actuators and 3 sensor \\
$\mathrm{A}_{5,4,3}-\mathrm{S}_{4,3,5}$ & Structure with 3 actuators and 4 sensor \\
$\mathrm{A}_{5,4,3}-\mathrm{S}_{4,3,5,2}$ & Structucture with 3 actuators and 5 sensor \\
$\mathrm{A}_{5,4,3}-\mathrm{S}_{4,3,5,2,1}$ & Structure
\end{tabular}

According to the result of Table 5, in the case of using one actuator and one sensor, the displacement of upper floors can be reduced to about $65 \%$. If the structural vibrations are controlled by using two actuators and one sensor the displacement of upper floors can be diminished about $65 \%$. Moreover, the mentioned reduction rate will reach to $75 \%$ in the case of three actuators and two sensors. If three sensors and actuators are used, the maximum displacement in fourth and fifth floor will be reduced to about 80 and 75 percent, respectively. Another factor, utilized for the numerical evaluation of the proposed method is the maximum kinetic energy
TABLE 5: The maximum structural displacements for the harmonic load.

\begin{tabular}{lccccc}
\hline \multirow{2}{*}{ Control case } & \multicolumn{5}{c}{ Maximum displacement $(\mathrm{cm})$} \\
& Floor 1 & Floor 2 & Floor 3 & Floor 4 & Floor 5 \\
\hline Control off & 1.215 & 2.288 & 3.368 & 3.939 & 3.912 \\
$\mathrm{~A}_{5}-\mathrm{S}_{4}$ & 0.414 & 0.769 & 1.139 & 1.301 & 1.305 \\
$\mathrm{~A}_{5,4}-\mathrm{S}_{4}$ & 0.388 & 0.666 & 0.983 & 1.161 & 1.125 \\
$\mathrm{~A}_{5,4,3}-\mathrm{S}_{4}$ & 0.44 & 0.846 & 1.137 & 1.314 & 1.373 \\
$\mathrm{~A}_{5,4,3}-\mathrm{S}_{4,3}$ & 0.284 & 0.522 & 0.776 & 0.96 & 1.335 \\
$\mathrm{~A}_{5,4,3}-\mathrm{S}_{4,3,5}$ & 0.217 & 0.417 & 0.553 & 0.756 & 0.983 \\
$\mathrm{~A}_{5,4,3}-\mathrm{S}_{4,3,5,2}$ & 0.173 & 0.322 & 0.498 & 0.719 & 0.989 \\
\hline
\end{tabular}

TABle 6: The maximum kinetic energy for the five-story shear building subjected to the harmonic load.

\begin{tabular}{lc}
\hline Control case & Maximum kinetic energy \\
\hline Control off & 548.14 \\
$\mathrm{~A}_{5}-\mathrm{S}_{4}$ & 54 \\
$\mathrm{~A}_{5,4}-\mathrm{S}_{4}$ & 41.19 \\
$\mathrm{~A}_{5,4,3}-\mathrm{S}_{4}$ & 56.51 \\
$\mathrm{~A}_{5,4,3}-\mathrm{S}_{4,3}$ & 29.94 \\
$\mathrm{~A}_{5,4,3}-\mathrm{S}_{4,3,5}$ & 15.74 \\
$\mathrm{~A}_{5,4,3}-\mathrm{S}_{4,3,5,2}$ & 12.66 \\
\hline
\end{tabular}

TABLE 7: The story drift of the fifth floor for the five-story shear building subjected to the harmonic load.

\begin{tabular}{lc}
\hline Control case & Story drift \\
\hline Control off & 2.69 \\
$\mathrm{~A}_{5}-\mathrm{S}_{4}$ & 0.912 \\
$\mathrm{~A}_{5,4}-\mathrm{S}_{4}$ & 0.820 \\
$\mathrm{~A}_{5,4,3}-\mathrm{S}_{4}$ & 0.993 \\
$\mathrm{~A}_{5,4,3} \mathrm{~S}_{4,3}$ & 1.118 \\
$\mathrm{~A}_{5,4,3}-\mathrm{S}_{4,3,5}$ & 0.777 \\
$\mathrm{~A}_{5,4,3}-\mathrm{S}_{4,3,5,2}$ & 0.852 \\
\hline
\end{tabular}

of structural dynamic analysis. Table 6 shows the maximum kinetic energy of fifth-story shear building subjected to the harmonic load. Accordingly, the proposed control approach has suitable performance in reducing the kinetic energy of the system so that by increasing number of sensors and actuators the kinetic energy is reduced considerably. Also, the variation of the fifth-story drift is different in various control cases, inserted in Table 7. It is clear that the performance of the proposed method increases by using more numbers of actuators.

To assess the proposed algorithm against the broadband earthquake excitations, this structure is analyzed for the seismic load, that is, the Elcentro earthquake accelerograms. The maximum displacements of the fourth and fifth floors, caused by the El Centro Earthquake, have been inserted in Table 8. Results of Table 8 show that if the structural vibration is controlled by one actuator and one sensor, the displacements of upper floors are reduced to about $42 \%$. 
TABLE 8: The maximum displacement of the fourth and fifth floors in the five-story shear building excited by the Elcentro earthquake.

\begin{tabular}{lcc}
\hline Case of control & \multicolumn{2}{c}{ Maximum displacement $(\mathrm{cm})$} \\
& Floor 4 & Floor 5 \\
\hline Control off & 8.11 & 9.2 \\
$\mathrm{~A}_{5}-\mathrm{S}_{4}$ & 3.94 & 4.66 \\
$\mathrm{~A}_{5,2,3}-\mathrm{S}_{4}$ & 3.93 & 4.6 \\
$\mathrm{~A}_{5,2,3}-\mathrm{S}_{4,3}$ & 3.108 & 3.88 \\
$\mathrm{~A}_{5,2,3}-\mathrm{S}_{4,3,5}$ & 2.4 & 2.4 \\
\hline
\end{tabular}

TABLE 9: The actuator and sensor locations for seven-story shear building.

\begin{tabular}{lcc}
\hline Degree of freedom & $\begin{array}{c}\text { Priority of the } \\
\text { actuator installation }\end{array}$ & $\begin{array}{c}\text { Priority of the sensor } \\
\text { installation }\end{array}$ \\
\hline 1 & 4 & 6 \\
2 & 2 & 4 \\
3 & 6 & 2 \\
4 & 1 & 1 \\
5 & 5 & 3 \\
6 & 3 & 5 \\
7 & 7 & 7 \\
\hline
\end{tabular}

TABLE 10: The maximum displacements of the seven-story shear building excited by the Elcentro earthquake.

\begin{tabular}{lcc}
\hline Case of control & \multicolumn{2}{c}{ Maximum displacement $(\mathrm{cm})$} \\
& Floor 6 & Floor 7 \\
\hline Control off & 12.4 & 13.2 \\
$\mathrm{~A}_{4}-\mathrm{S}_{4}$ & 9.2 & 9.7 \\
$\mathrm{~A}_{4,2}-\mathrm{S}_{4,3}$ & 8 & 8.4 \\
\hline
\end{tabular}

Moreover, the mentioned reduction will reach to about $70 \%$ if three actuators and two sensors are used.

Figure 5 shows the time history displacement of the fifthstory for different control processes. Moreover, the variation of the fifth floor acceleration for both controlled and controloff system has been plotted in Figure 6. Based on Figures 5 and 6 the proposed algorithm, presented for determining the locations of the sensor and actuator and also calculating the actuator's force, has a suitable applicability in numerical results.

Moreover, the time history for both of the earthquake and the actuator forces (for the case of one actuator and one sensor) have been plotted in Figure 7 . It is clear that the maximum actuators force is less than fifty percent of the floors weight. Moreover, Figure 7 shows that there is logical balance between the actuator's force and the earthquake load.

5.3. Seven-Story Shear Building. Here, a seven-story shear building is analyzed so that the lumped mass and lateral stiffness of each story are $0.2591 \mathrm{~kg}$ and $100 \mathrm{~N} / \mathrm{cm}$, respectively. This structure is excited by the Elcentro acceleration. To control the vibration of the structure, the sensors and

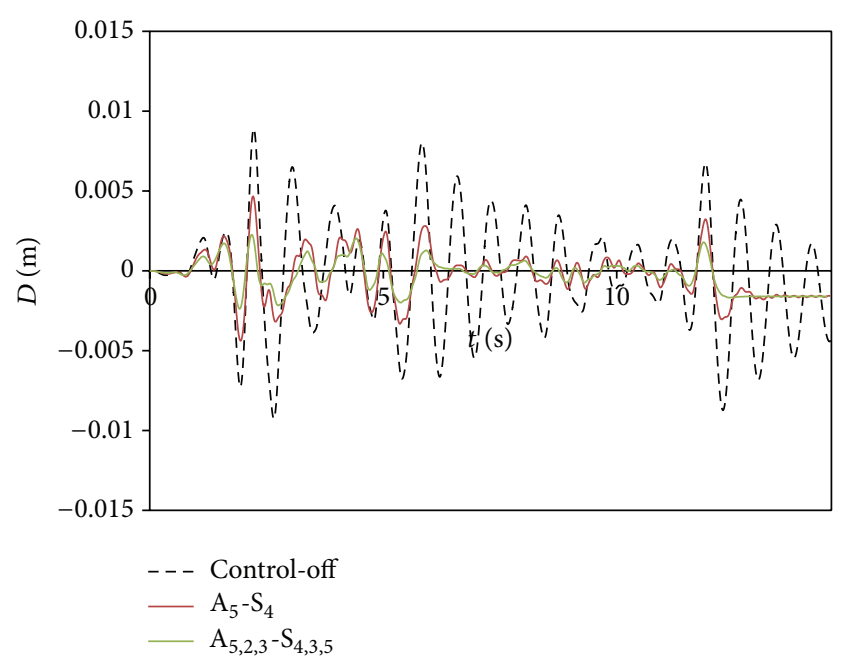

FIGURE 5: The time history displacement for the fifth-story of shear building excited by the Elcentro earthquake.

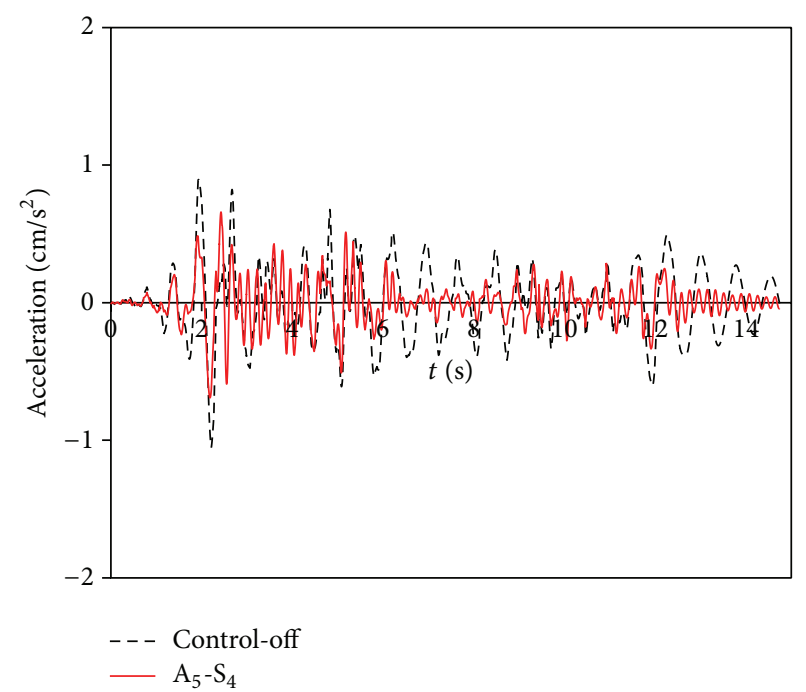

FIGURE 6: The time history acceleration for the fifth-story of shear building excited by the Elcentro earthquake.

actuators locations are determined based on the proposed algorithm. The details of such procedure have been inserted in Table 9. Using the various control algorithms with regard to the number of actuators and sensors, the maximum displacements of the sixth and seventh floors have been inserted in Table 10.

Results demonstrate that the proposed control approach is suitable for reducing the maximum displacements of the system. Moreover, the time history displacement of the top of the structure has been plotted in Figure 8 for different control cases. Figure 8 shows that the efficiency of the proposed active control scheme increases if more number of sensor and actuator is used.

All of the above dynamic analyses are completely stable so that any instability in the control process of the proposed algorithm is not considered. It is worth emphasizing that 


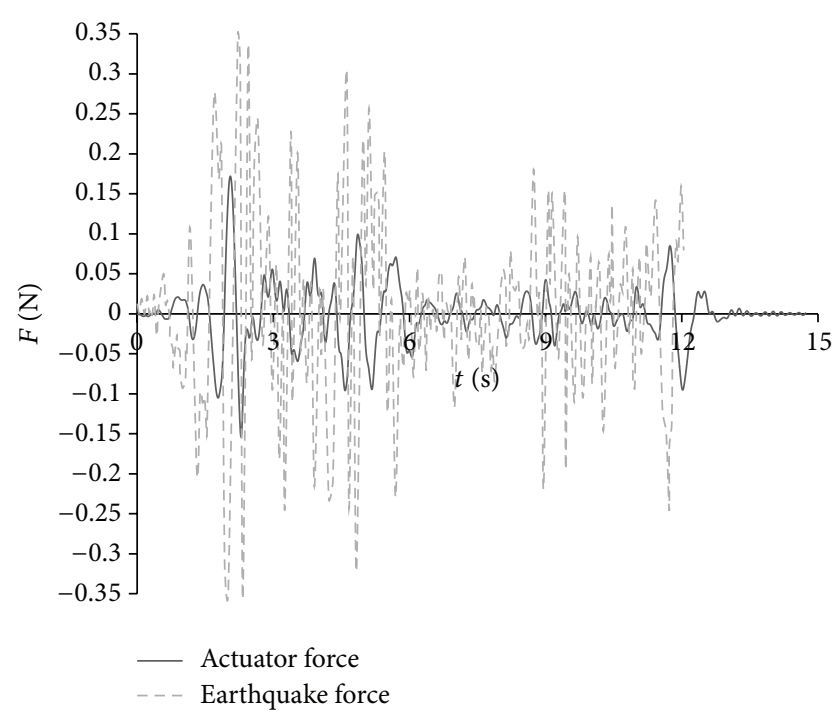

FIgURE 7: Comparison between the actuator and the earthquake forces in the case of using one actuator and one sensor.

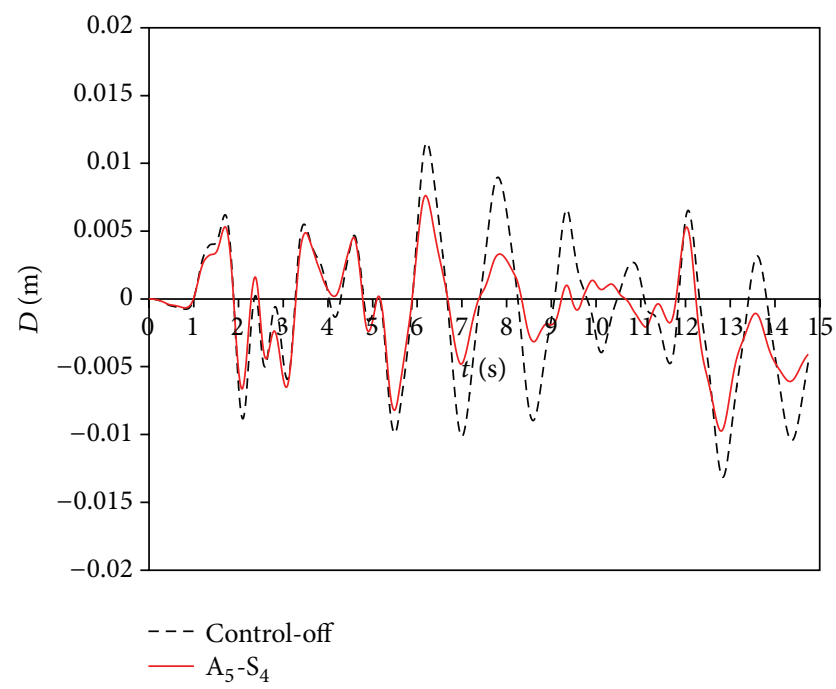

FIGURE 8: The time history displacement for the highest floor of the seven-story shear building excited by the Elcentro earthquake.

the proposed active control mechanism does not impose any additional condition for stability of the analysis, because it is formulated based on the well-known structural dynamic theories. From this point of view, the prepared algorithm has suitable compatibility with dynamic behavior of structures. This subject reduces the instability potential that exists in any active control method.

\section{The Effect of Uncertainty in the Proposed Method}

There are many variables in the analysis and design of structures such as loads, capacity of elements, and material properties. Uncertainty in each of these variables has a significant effect on structure safety. Stories mass in this section are considered as random variables. To evaluate the uncertainty of stories mass in the proposed method, for each story, we considered a range of numbers.

In the range of $+15 \%$ and $-15 \%$, a set of random numbers is generated by MATLAB program as stories masses. In this case, the stories masses in each of the cases considered purely accidental (casel until case 30) and each story may be reduced or increased compared to the base masses. Table 11 shows the results of the proposed method using a sensor and an actuator, in various states of random masses in the range of 15 percent compared to the base masses. According to the received results, changes in masses in the range of $15 \%$ caused displacement increment up to $14.6 \%$ and displacement reduction up to $10 \%$ for fourth floor. Also, in this condition, the displacement of fifth floor, increased up to $18 \%$ and decreased up to $14 \%$.

\section{Applicability of the Proposed Method}

In the case of applicability of the proposed method in realistic structures, using of the piezoelectric stack could be useful for a lot of reasons. In this way the method which has been introduced by Kamada et al. [7] is proposed. In this method piezoelectric stack actuators are placed at the bottom of firststory columns in both sides. If the induced voltage is applied in reverse phase to these actuators, the concentrated bending moments $M_{a}$ are produced at the bottom of columns. By applying the basic structural calculations, the equivalent shear force at each story level is determined as follows:

$$
\begin{gathered}
M_{a}=F_{p} r, \\
F_{a}=\frac{24 \mathrm{hr}}{L 3}(L-2 H) F_{p},
\end{gathered}
$$

where $L, F_{p}$, and $F_{a}$ are the columns height, actuators produced force per unite voltage, and equivalent shear force produced by actuators in first floor level, respectively. Other parameters have been shown in Figure 9.

\section{Conclusions}

In this paper, a new method was developed for active control of structure. This process was based on the theory of structural dynamics. In the proposed model, the structural fluctuations are controlled by multiple actuators and sensors and the actuator has been modeled as an additional viscous damper. In this way, the actuators forces are calculated in such a way that a greater number of low structural damping of the various modes of vibration is critical. Actuators forces are calculated in such a way that more first equivalent modal coordinates damping is critical. To consider the proposed method, the changes in maximum displacement, the maximum kinetic energy, and total maximum relative displacement of stories were considered in various control cases in a five-story structure. Based on the numerical results, in the case of using an actuator and one sensor, the displacement 
TABLE 11: The effect of uncertainty on fourth and fifth floor displacement.

\begin{tabular}{|c|c|c|}
\hline Analysis case & $\begin{array}{c}\text { Fourth floor } \\
\text { displacement }(\mathrm{cm})\end{array}$ & $\begin{array}{c}\text { Fifth floor } \\
\text { displacement }(\mathrm{cm})\end{array}$ \\
\hline Control off & 3.939 & 3.912 \\
\hline $\mathrm{A}_{5}-\mathrm{S}_{4}$ (base masses) & 1.301 & 1.305 \\
\hline Case 1 & 1.38 & 1.39 \\
\hline Case 2 & 1.491 & 1.544 \\
\hline Case 3 & 1.276 & 1.266 \\
\hline Case 4 & 1.249 & 1.251 \\
\hline Case 5 & 1.216 & 1.192 \\
\hline Case 6 & 1.360 & 1.383 \\
\hline Case 7 & 1.269 & 1.248 \\
\hline Case 8 & 1.321 & 1.332 \\
\hline Case 9 & 1.455 & 1.467 \\
\hline Case 10 & 1.384 & 1.427 \\
\hline Case 11 & 1.386 & 1.396 \\
\hline Case 12 & 1.261 & 1.280 \\
\hline Case 13 & 1.328 & 1.334 \\
\hline Case 14 & 1.296 & 1.280 \\
\hline Case 15 & 1.339 & 1.363 \\
\hline Case 16 & 1.271 & 1.245 \\
\hline Case 17 & 1.408 & 1.446 \\
\hline Case 18 & 1.280 & 1.2796 \\
\hline Case 19 & 1.260 & 1.246 \\
\hline Case 20 & 1.228 & 1.202 \\
\hline Case 21 & 1.343 & 1.377 \\
\hline Case 22 & 1.393 & 1.411 \\
\hline Case 23 & 1.374 & 1.389 \\
\hline Case 24 & 1.213 & 1.192 \\
\hline Case 25 & 1.364 & 1.387 \\
\hline Case 26 & 1.214 & 1.179 \\
\hline Case 27 & 1.275 & 1.257 \\
\hline Case 28 & 1.170 & 1.122 \\
\hline Case 29 & 1.429 & 1.440 \\
\hline Case 30 & 1.310 & 1.310 \\
\hline
\end{tabular}

of upper floors can be reduced to about $65 \%$ compared to the case of lack of control system. Also, the displacement in upper stories reduced in the case of using two actuators and one sensor. Moreover, in the case of three actuators and two sensors at the proper locations the maximum upper stories displacement reduced up to $75 \%$. In term of maximum kinetic energy, using one actuator and one sensor reduced this item up to $90 \%$. Beside, using three actuators and sensors caused the total maximum relative displacement of stories to diminish up to $70 \%$. In addition, the uncertainty effect of the mass of stories on the floor's displacement was investigated.

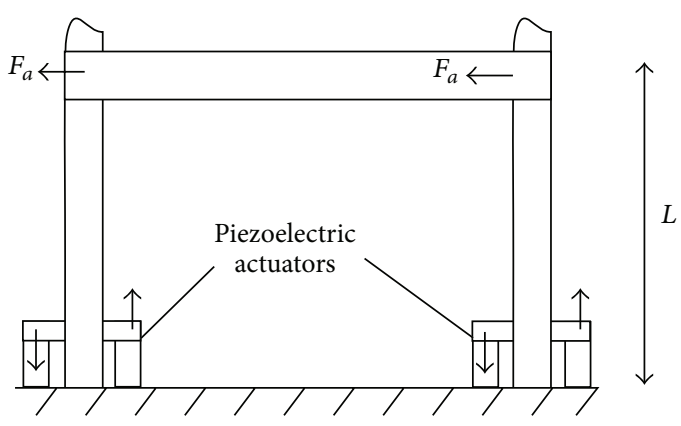

(a)

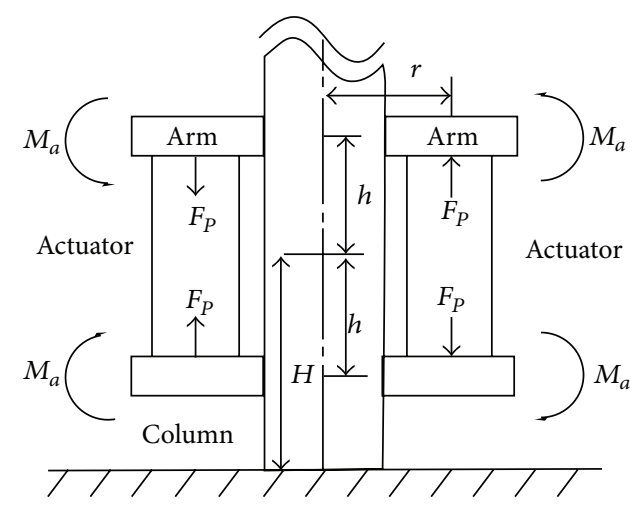

(b)

FIGURE 9: The piezoelectric actuator's mechanism (a) front view and (b) side view.

\section{Conflict of Interests}

The authors declare that there is no conflict of interests regarding the publication of this paper.

\section{References}

[1] S. Akutagawa, M. Otsuki, and Y. Kitagawa, "Hybrid control system with optimal fuzzy logic and genetic algorithm for highrise buildings," in Proceedings of the 13th World Conference on Earthquake Engineering, Vancouver, Canada, 2004.

[2] R. Guclu and H. Yazici, "Vibration control of a structure with ATMD against earthquake using fuzzy logic controllers," Journal of Sound and Vibration, vol. 318, no. 1-2, pp. 36-49, 2008.

[3] G. Chen and J. Wu, "Optimal placement of multiple tune mass dampers for seismic structures," Journal of Structural Engineering, vol. 127, no. 9, pp. 1054-1062, 2001.

[4] F. Rüdinger, "Tuned mass damper with nonlinear viscous damping," Journal of Sound and Vibration, vol. 300, no. 3-5, pp. 932-948, 2007.

[5] N. Hoang, Y. Fujino, and P. Warnitchai, "Optimal tuned mass damper for seismic applications and practical design formulas," Engineering Structures, vol. 30, no. 3, pp. 707-715, 2008.

[6] D. S. Bayard, F. Y. Hadaegh, and D. R. Meldrum, "Optimal experiment design for identification of large space structures," Automatica, vol. 24, no. 3, pp. 357-364, 1988.

[7] T. F. Kamada, T. Fujita, T. Hatayama et al., "Active vibration control of flexural-shear type frame structures with smart structures using piezoelectric actuators," Smart Materials and Structures, vol. 7, no. 4, pp. 479-488, 1998. 
[8] J.-H. Han and I. Lee, "Optimal placement of piezoelectric sensors and actuators for vibration control of a composite plate using genetic algorithms," Smart Materials and Structures, vol. 8, no. 2, pp. 257-267, 1999.

[9] A. M. Sadri, J. R. Wright, and R. J. Wynne, "Modelling and optimal placement of piezoelectric actuators in isotropic plates using genetic algorithms," Smart Materials and Structures, vol. 8, no. 4, pp. 490-498, 1999.

[10] F. Gao, Y. Shen, and L. Li, "Optimal design of piezoelectric actuators for plate vibroacoustic control using genetic algorithms with immune diversity," Smart Materials and Structures, vol. 9, no. 4, pp. 485-491, 2000.

[11] H. Zhang, B. Lennox, P. R. Goulding, and A. Y. T. Leung, "Float-encoded genetic algorithm technique for integrated optimization of piezoelectric actuator and sensor placement and feedback gains," Smart Materials and Structures, vol. 9, no. 4, pp. 552-557, 2000.

[12] Z.-J. Cao, B.-C. Wen, and G.-W. Meng, "Topological optimization of placements of the piezoelectric actuator of intelligent structures," Journal of Northeastern University, vol. 21, no. 4, pp. 383-385, 2000.

[13] M. Kögl and E. C. N. Silva, "Topology optimization of smart structures: design of piezoelectric plate and shell actuators," Smart Materials and Structures, vol. 14, no. 2, pp. 387-399, 2005.

[14] V. Sethi and G. Song, "Multimode vibration control of a smart model frame structure," Smart Materials and Structures, vol. 15, no. 2, pp. 473-479, 2006.

[15] J. V. Song, S. E. Johnson, and B. N. Agrawal, "Ambient active vibration control of a space truss using a lead zirconate stack actuator," in Proceedings of the Institution of Mechanical Engineers, vol. 215, p. 355, 2001.

[16] T. K. Datta, "A state of the art review on active control of structures," 22nd ISET Annual Lecture, ISET Journal of Earthquake Technology, Paper no. 430, vol. 40, no. 1, pp. 1-17, 2003.

[17] D. S. Michael and C. C. Michael, "Semi-active control systems for seismic protection of structures: a state-of-the-art review," Engineering Structures, vol. 21, no. 6, pp. 469-487, 1999.

[18] J.-N. Yang, "Application of optimal control theory to civil engineering structures," Journal of Engineering Mechanics Division, vol. 101, no. 6, pp. 819-838, 1975.

[19] M. Abdel-Rohman and H. H. Leipholz, "Active control of flexible structures," Journal of Structural Division, vol. 104, no. 8, pp. 1251-1266, 1978.

[20] L. Meirovitch and H. Oz, "Modal space control of distributed gyroscopic systems," Journal of Guidance, Control, and Dynamics, vol. 6, pp. 20-25, 1980.

[21] J. N. Yang, A. Akbarpour, and P. Ghaemmaghami, "New optimal control algorithms for structural control," Journal of Engineering Mechanics, vol. 113, no. 9, pp. 1369-1386, 1987.

[22] A. M. Reinhorn, G. D. Manolis, and C. Y. Wen, "Active control of inelastic structures," Journal of Engineering Mechanics, vol. 113, no. 3, pp. 315-333, 1987.

[23] Z. Wu, R. C. Lin, and T. T. Soong, "Non-linear feedback control for improved peak response reduction," Smart Materials and Structures, vol. 4, no. 1, pp. A140-A148, 1995.

[24] J. N. Yang, Z. Li, and S. C. Liu, "Instantaneous optimal control with acceleration and velocity feedback," Probabilistic Engineering Mechanics, vol. 6, no. 3-4, pp. 204-211, 1991.

[25] J. N. Yang, J. C. Wu, A. K. Agrawal, and Z. Li, "Sliding mode control for seismic-excited linear and non-linear civil engineering structures," Technical Report NCEER 94-0017, 1994.
[26] M. Abdel-Rohman, "Structural control considering time-delay effect," Transactions of the Canadian Society for Mechanical Engineering, vol. 9, no. 4, pp. 224-227, 1985.

[27] A. Tani, H. Kawamura, and S. Ryu, "Intelligent fuzzy optimal control of building structures," Engineering Structures, vol. 20, no. 3, pp. 184-192, 1998.

[28] M. H. Korayem and A. Alipour, "Dynamic analysis of moving cables with variable tension and variable speed," International Journal of Engineering, Transactions B: Applications, vol. 23, no. 2, pp. 157-168, 2010.

[29] J. Alamatian and J. Rezaeepazhand, "A simple approach for determination of actuator and sensor locations in smart structures subjected to the dynamic loads," International Journal of Engineering, Transactions A, vol. 24, no. 4, pp. 341-349, 2011.

[30] V. D. Rao, Matlab an Introduction and Applications, New Age Publishers, 2010.

[31] R. W. Clough and J. Penzien, Dynamics of Structures, McGraw Hill, New York, NY, USA, 1993. 

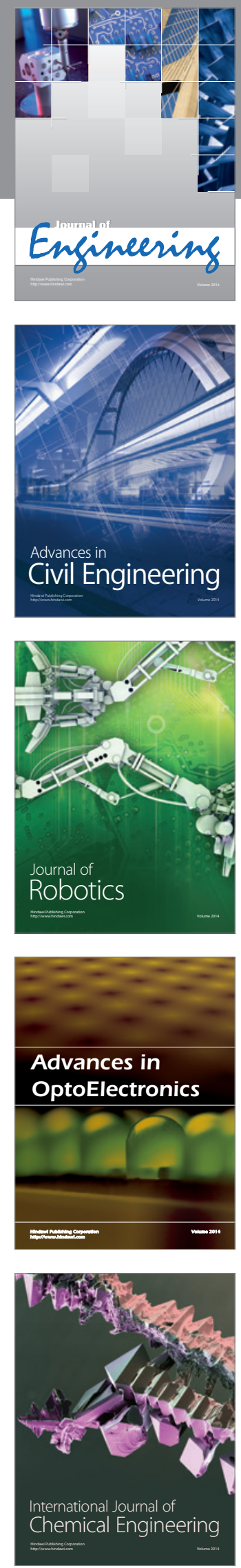

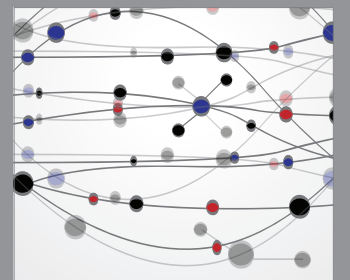

The Scientific World Journal
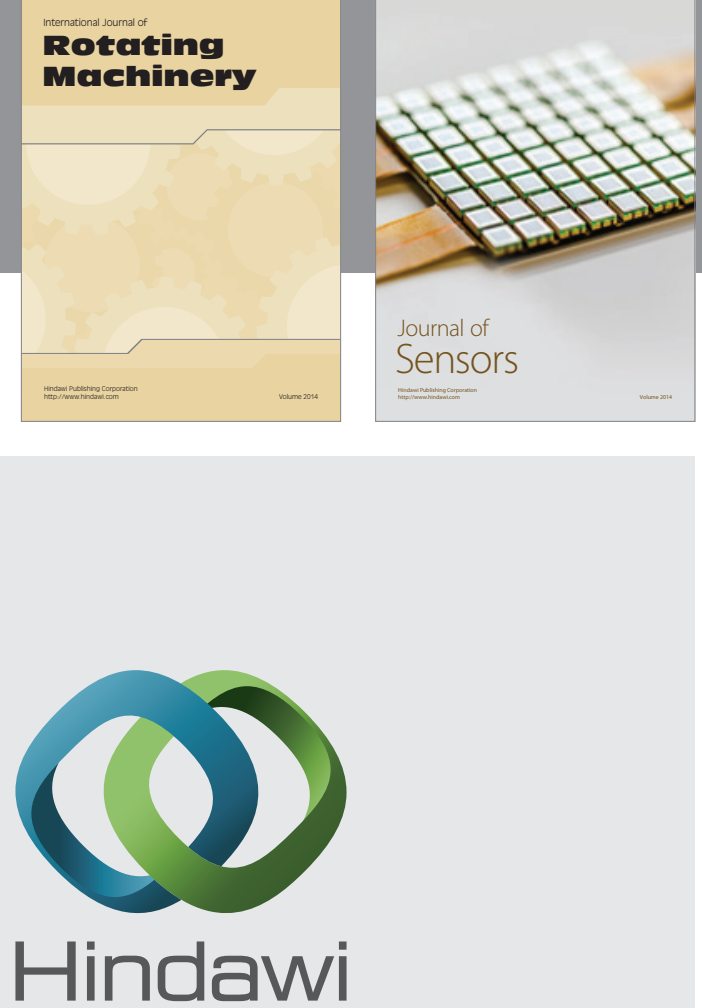

Submit your manuscripts at http://www.hindawi.com
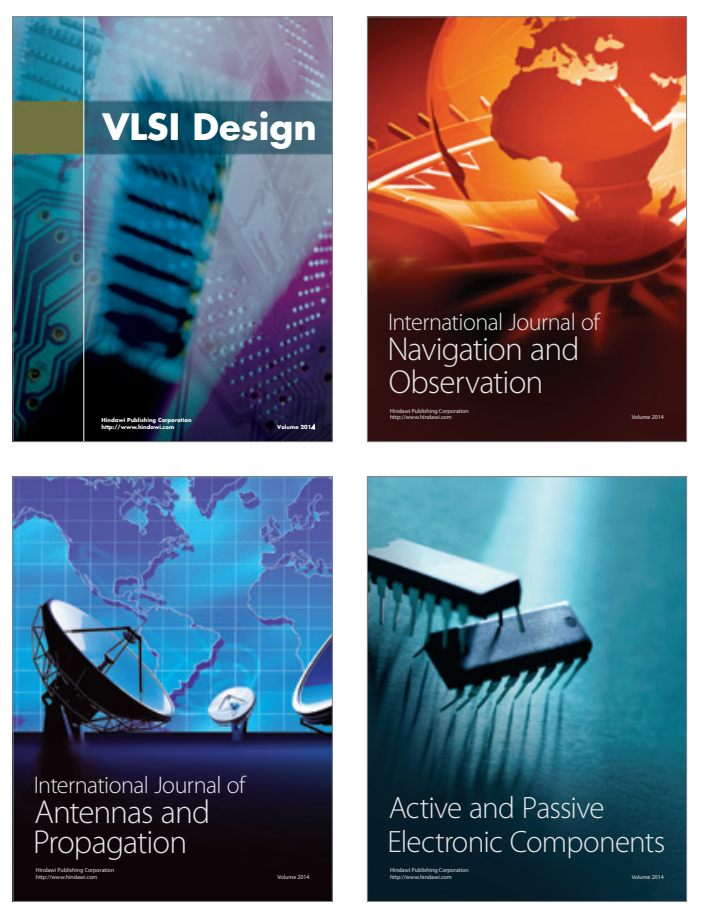
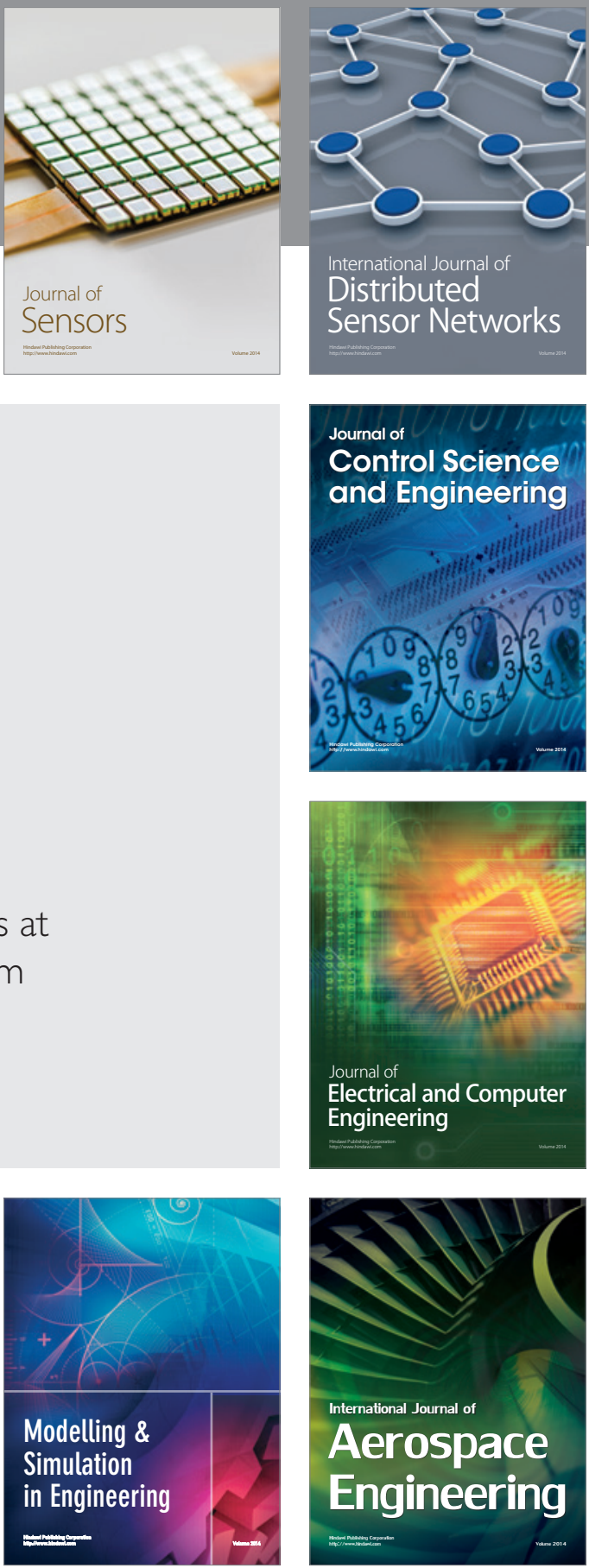

Journal of

Control Science

and Engineering
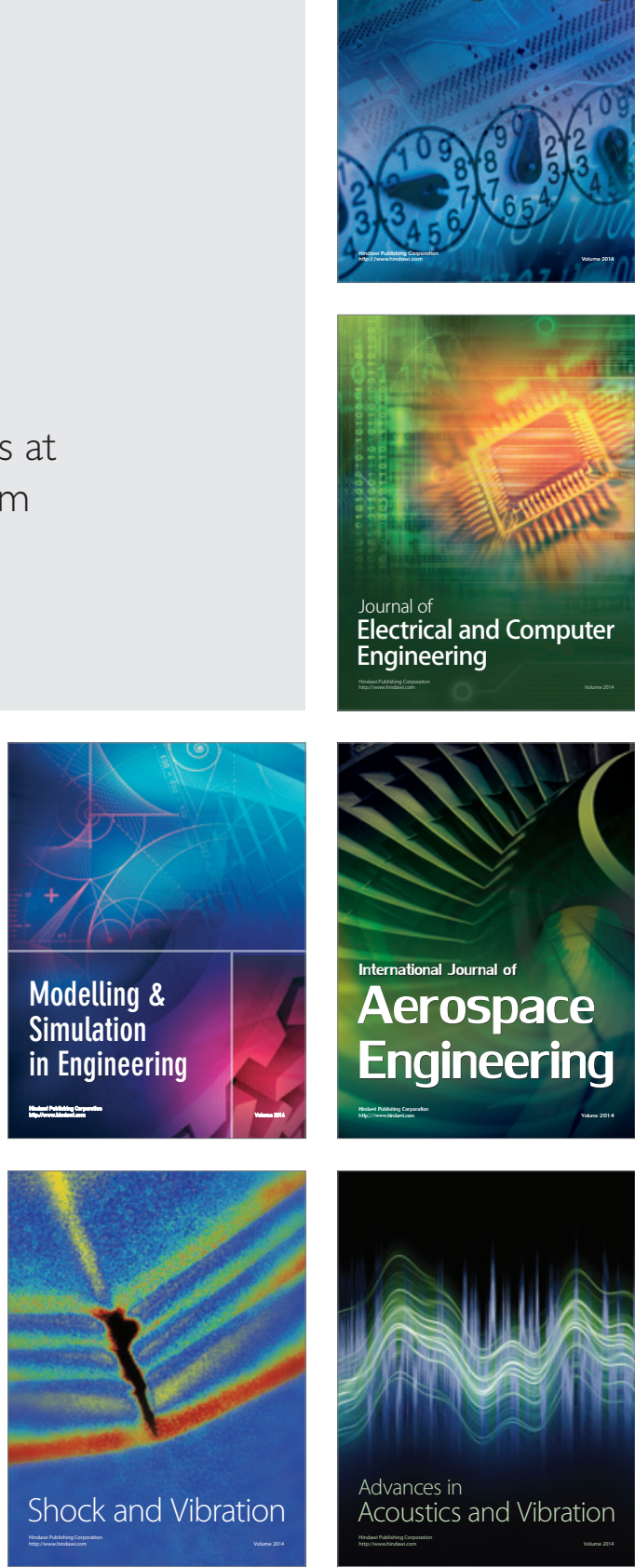